\title{
Retracted: Restoration of Critical-Sized Defects in the Rabbit Mandible Using Autologous Bone Marrow Stromal Cells Hybridized with Nano- $\beta$-tricalcium Phosphate/Collagen Scaffolds
}

\author{
Journal of Nanomaterials \\ Received 15 March 2015; Accepted 15 March 2015 \\ Copyright (C) 2015 Journal of Nanomaterials. This is an open access article distributed under the Creative Commons Attribution \\ License, which permits unrestricted use, distribution, and reproduction in any medium, provided the original work is properly \\ cited.
}

The paper titled "Restoration of Critical-Sized Defects in the Rabbit Mandible Using Autologous Bone Marrow Stromal Cells Hybridized with Nano- $\beta$-tricalcium Phosphate/Collagen Scaffolds" [1] has been retracted upon the authors' request, as they found that there are some wrong results in their paper.

\section{References}

[1] X. Zhang, M. Xu, X. Liu et al., "Restoration of critical-sized defects in the rabbit mandible using autologous bone marrow stromal cells hybridized with nano- $\beta$-tricalcium phosphate/ collagen scaffolds," Journal of Nanomaterials, vol. 2013, Article ID 913438, 8 pages, 2013. 


\title{
Restoration of Critical-Sized Defects in the Rabbit Mandible
} Using Autologous Bone Marrow Stromal Cells Hybridized with Nano- $\beta$-tricalcium Phosphate/Collagen Scaffolds

\author{
Xuehui Zhang, ${ }^{1,2}$ Mingming Xu, ${ }^{1}$ Xinggang Liu, ${ }^{1}$ Feng Zhang, ${ }^{3}$ Yan Wei, ${ }^{1}$ Song Meng, \\ Xiaohan Dai, ${ }^{1}$ Aizhu Duan, ${ }^{1}$ and Xuliang Deng ${ }^{1,4}$ \\ ${ }^{1}$ Department of Geriatric Dentistry, Peking University School and Hospital of Stomatology, Beijing 100081, China \\ ${ }^{2}$ State Key Laboratory of New Ceramics and Fine Processing, Department of Materials Science and Engineering, Tsinghua University, \\ Beijing 100084, China \\ ${ }^{3}$ The Oral Clinic of the 2nd Hospital of Beijing Armed Police Force, Beijing 100037, China \\ ${ }^{4}$ National Engineering Laboratory for Digital and Material Technology of Stomatology, Beijing 100081, China
}

Correspondence should be addressed to Xuliang Deng; kqdengxuliang@bjmu.edu.cn

Received 26 July 2013; Accepted 26 September 2013

Academic Editor: Haifeng Chen

Copyright ( 2013 Xuehui Zhang et al. This is an open access article distributed under the Creative Commons Attribution License, which permits unrestricted use, distribution, and reproduction in any medium, provided the original work is properly cited.

Nano- $\beta$-tricalcium phosphate/collagen $(\mathrm{n}-\beta-\mathrm{TCP} / \mathrm{Col})$ is considered with good osteoconductivity. However, the therapeutic effectiveness of $\mathrm{n}-\beta$-TCP/Col scaffolds in combination with autologous bone marrow stromal cells (BMSCs) remains to be elucidated for the repair of critical-sized bone defects. In this study, we found that $\mathrm{n}$ - $\beta$-TCP/Col scaffolds exhibited high biocompatibility in vitro. The introduction of BMSCs expanded in vitro to the scaffolds dramatically enhanced their efficiency to restore critical-sized bone defects, especially during the initial stage after implantation. Collectively, these results suggest that autologous BMSCs in $\mathrm{n}-\beta$-TCP/Col scaffolds have the potential to be applied in bone tissue engineering.

\section{Introduction}

In recent years, a critical-sized bone defect has been defined as an intraosseous wound that will not spontaneously heal completely without surgical intervention $[1,2]$. Bone autografting is currently considered to be the "gold standard" for clinical treatment. However, it is always associated with irregular rates of resorption, pain, and morbidity of the donor site, and it requires additional surgical procedures [3]. Therefore, alternatives to autologous bone grafting are the subject of intensive research.

Recently, tissue engineering approaches have proven to be very effective in bone regeneration. Successful repair of bone defects has been investigated in many animal species including mice [4], rats [5, 6], rabbits [7], canines [8], and goats [9]. Selection of the most appropriate scaffold for application in bone tissue engineering is a critically important step towards tissue-engineered product $[10,11]$. $\beta$-Tricalcium phosphate $(\beta$-TCP) is a bioactive and biodegradable ceramic that has been widely used as a scaffold for bone repair because of its excellent biocompatibility, reabsorbability, and osteoconductive properties [12,13]. However, the use of $\beta$ TCP is limited because of its brittleness and low plasticity [14]. Biocompatible polymers, such as collagen ( $\mathrm{Col})$, are also regarded as applicable candidates for use in bone regeneration scaffolds. However, there are a number of practical problems for their use alone, including an uncontrollable degradation rate in vivo and poor mechanical properties compared with those of natural hard tissues $[15,16]$.

To overcome the limitations of bioceramics and biopolymers, ceramic/polymer composite materials have been explored for preparation of bone tissue engineering scaffolds $[17,18]$. Using a biomimetic strategy, composites based on $\beta$ TCP and Col have been developed with a three- dimensional structure to fill bone defects, which show promise because of compositional and structural analogies to natural bone [1921]. In our previous study [22], we designed and fabricated a novel three-dimensional porous tissue engineering scaffolds 
using nanosized $\beta$-TCP (n- $\beta$-TCP)/Col composite materials. The $\mathrm{n}-\beta$-TCP particles (about $300 \mathrm{~nm}$ ) can be distributed homogenously in the skeleton of a collagen fibril network in which $\mathrm{n}-\beta$-TCP particles bind tightly to collagen fibrils. These composite scaffolds have been found to repair small bone defects. However, the therapeutic effectiveness of $n-\beta$ $\mathrm{TCP} / \mathrm{Col}$ scaffolds in combination with seed cells remains to be elucidated for the repair of critical-sized bone defects.

Bone marrow stromal cells (BMSCs) are multipotent and have the ability to differentiate into osteoblastic, chondrocytic, and adipocytic lineage cells. BMSCs exhibit advantages over other cell types and have been wildly used in bone tissue engineering [23]. However, isolation of bone marrow has some disadvantages in clinical practice, especially to obtain sufficient cell numbers for transplantation into large bone defects. To obtain enough cell numbers, much bone marrow aspiration is needed which would lead to an invasive and painful procedure and increase the risk of morbidity and infection. Expansion of BMSCs in vitro is an alternative strategy to gain enough cells for implantation.

The goal of this study was to explore the therapeutic effectiveness of in vitro expanded autologous BMSC-loaded n$\beta$-TCP/Col scaffolds for repairing critical-sized bone defects of the rabbit mandible. At 4 and 8 weeks after implantation, gross examination, cone beam computed tomography ( $\mathrm{CBCT}$ ) scanning, and histological analysis were used to assess new bone formation and scaffolds degradation.

\section{Materials and Methods}

2.1. Fabrication and Characterization of $n-\beta-T C P / C o l$ Scaffolds. $\beta$-TCP $(\sim 300 \mathrm{~nm}$ particle size $)$ was synthesized by calcining an amorphous calcium phosphate precursor [24]. $\mathrm{n}-\beta$-TCP/Col scaffolds were prepared as reported in our previous study [25]. Briefly, type I collagen (Sigma-Aldrich Co., USA) was dispersed in a diluted hydrochloric acid solution ( $\mathrm{pH} 2)$ at room temperature. $\beta$-TCP was added to the collagen suspension with stirring at a $3: 1(\mathrm{w} / \mathrm{w})$ ratio. After formation of a uniform suspension, the crossing-linking reagent glutaraldehyde (Shanghai Pharm Co., China) was added to the mixture. After freezing in liquid nitrogen, the mixture was lyophilized to obtain the porous $\mathrm{n}-\beta$-TCP/Col composite. After soaking in deionized water for 4 days with daily changes to remove unreacted glutaraldehyde, the porous composite was lyophilized again to form the $n-\beta$ TCP/Col composite scaffolds.

The surface morphology and structure of the nanofibers were observed by scanning electron microscopy (SEM, S-4700; Hitachi, Japan). Fourier-transform infrared spectroscopy (FT-IR, 8700; Nicolet, USA) was used to evaluate the chemical structure of the composite scaffolds.

2.2. Isolation, Characterization, and Osteogenic Differentiation of Rabbit BMSCs. All animal experiments were performed in accordance with a protocol approved by the Laboratory Animal Care and Use Committee of Peking University. All reagents were purchased from Sigma-Aldrich Co. (St. Louis, MO) unless otherwise stated. BMSCs were isolated from bone marrow aspirates of New Zealand white rabbits (12 weeks old, $2.5-3 \mathrm{~kg}, n=12$ ). Briefly, the bone marrow aspirate was washed three times with Hanks' balanced salt solution, plated onto $100 \mathrm{~mm}$ dishes, and cultured in Dulbecco's modified Eagle's medium (DMEM) (Gibco, USA) supplemented with $10 \%$ fetal bovine serum (FBS) (HyClone, USA), $100 \mathrm{U} / \mathrm{mL}$ penicillin, and $100 \mathrm{mg} / \mathrm{mL}$ streptomycin at $37^{\circ} \mathrm{C}$ with $5 \% \mathrm{CO}_{2}$. After 72 hours, nonadherent cells were removed by changing the medium. To characterize the surface marker expression of BMSCs, passage 3 cells were stained with an anti-rabbit CD34 antibody (1:200; Abcam, Cambridge, MA) for immunohistological analysis (Figure 1(b)). At 80-90\% confluence, adherent passage 3 cells were trypsinized, harvested, and subcultured in osteogenic medium. The osteogenic medium consisted of DMEM supplemented with $10 \%$ FBS, $50 \mathrm{mg} / \mathrm{mL}$ ascorbic acid-2-phosphate, $100 \mathrm{nM}$ dexamethasone, $10 \mathrm{mM}$ $\beta$-glycerophosphate, $100 \mathrm{U} / \mathrm{mL}$ penicillin, and $100 \mathrm{mg} / \mathrm{mL}$ streptomycin. After 1 week of culture, the osteogenic differentiation of BMSCs was confirmed by alkaline phosphatase (ALP) staining.

2.3. Growth and Bioactivity of Rabbit BMSCs in n- $\beta-T C P / C o l$ Scaffolds. The fabricated $\mathrm{n}-\beta$-TCP/Col scaffolds $(10 \times 8 \times$ $3 \mathrm{~mm}^{3}$ ) were sterilized with ethylene oxide and prewetted in osteogenic medium for $24 \mathrm{~h}$. BMSCs that had been cultured in osteogenic medium for 1 week were seeded in the prewetted $n-\beta$-TCP/Col scaffolds $\left(2 \times 10^{7}\right.$ cells/scaffold $)$, and then the cell/scaffold constructs were placed in 24 -well plates. The cell/scaffold constructs were incubated for 3 hours to allow cell attachment, and then an additional $1 \mathrm{~mL}$ of culture medium was added to each well. After 3 days of culture, the constructs were fixed in $2.5 \%$ glutaraldehyde and serially dehydrated in an increasing ethanol gradient, air-dried, and sputter coated with gold prior to imaging by SEM at $20 \mathrm{kV}$.

At 1,3 , and 7 days of culture, cell proliferation was assayed using a CCK-8 kit (Dojindo, Japan) and enzymelinked immunosorbent assay reader (Bio-Rad, Hercules, CA, USA) at an absorbance wavelength of $450 \mathrm{~nm}$. The ALP activity of the adherent cells was also assessed using an Alkaline Phosphatase Assay Kit (Abcam, Cambridge, MA, USA) according to the manufacturer's instructions.

2.4. Experimental Animal Model. Twelve healthy New Zealand white rabbits were divided into two groups, one group for implantation of $\mathrm{n}-\beta-\mathrm{TCP} / \mathrm{Col}$ scaffolds alone and the other for $\mathrm{n}-\beta-\mathrm{TCP} / \mathrm{Col}$ scaffolds combined with autologous BMSCs expanded in vitro. The rabbits were anesthetized with pentobarbital sodium and a $3 \mathrm{~cm}$ parallel incision was made along the inferior border of the mandible on both sides. The periosteum was then retracted to expose the submaxilla. A critical-sized defect, $10 \times 8 \times 3 \mathrm{~mm}^{3}$, was made in the Buccallingual direction on one side of the mandible of each rabbit. The scaffolds alone or MSC/scaffold constructs were inserted into the defected. The mucoperiosteal flaps were carefully sutured over the defect area using 3-0 absorbable sutures. The rabbits were sacrificed at 4 and 8 weeks after implantation for tissue processing. 


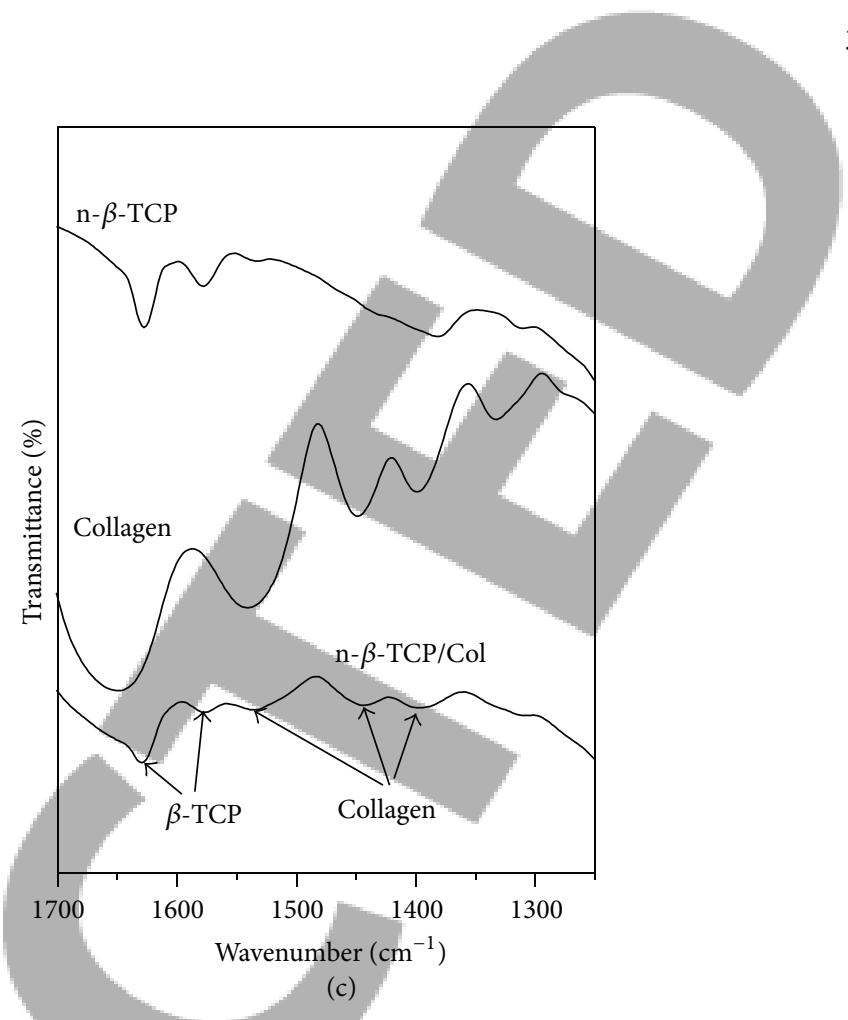

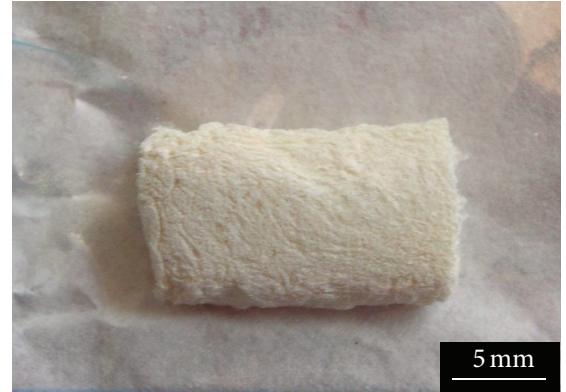

(a)

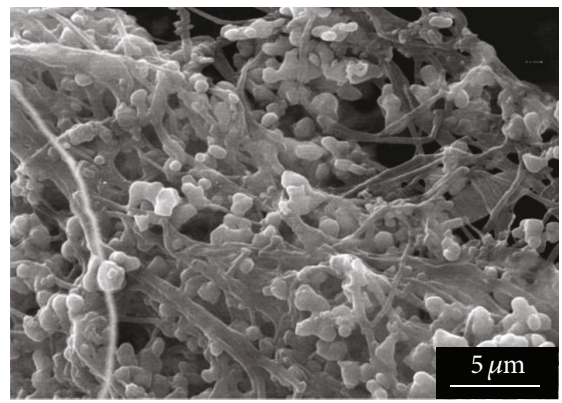

(b)

FIGURE 1: Characteristics of the $\mathrm{n}-\beta$-TCP/Col scaffolds. (a) Macroscopic image, (b) SEM image, and (c) FT-IR spectra of the n- $\beta$-TCP/Col
scaffolds. scaffolds.

2.5. Gross Observation and CBCT Examination. At 4 and 8 weeks after implantation, the scaffolds and surrounding tissues were excised and fixed in 10\% neutral buffered formalin. Each mandible defect site was assessed by gross observation, and the morphology of the reconstructed mandibles at 8 weeks after-implantation was assessed by CBCT scanning (J. Morita Mfg. Corp., Kyoto, Japan) at 60 KV and $2.0 \mathrm{~mA}$.

2.6. Histological Evaluation and Histomorphometry. At 4 and 8 weeks after implantation, rabbit mandibles were subjected to tissue processing and sectioning as reported previously [26]. Briefly, the tissue samples were fixed in $10 \%$ neutral buffered formalin for 7 days, followed by decalcification and dehydration, and finally embedded in paraffin and sectioned at $5 \mathrm{~mm}$ thicknesses. Hematoxylin and eosin (H\&E) staining was performed on serial tissue sections, and images were captured under a microscope (CX21; Olympus, Japan). For semiquantitative evaluation, histomorphometric analyses were performed according to a previously reported method [27] with slight modifications. The bone tissue area and residual scaffold materials area were measured using ImageJ software (National Institutes of Health, Bethesda, MD, USA) in three randomly selected areas $\left(1 \times 1 \mathrm{~mm}^{2}\right)$ per histological section. The bone area and residual scaffold materials area were expressed as the percentages of the total tissue area measured in each group.

2.7. Statistical Analysis. Quantitative data are presented as the mean \pm standard deviation. Statistical differences between groups were evaluated by the Student's $t$-test using SPSS 13.0 (SPSS Science). The critical level of statistical significance was set at $P<0.05$.

\section{Results and Discussion}

3.1. Physicochemical Properties of $n-\beta-T C P / C o l$ Scaffolds. Figure 1 shows the morphology and structure of $n-\beta$ TCP/Col scaffolds. The composite scaffolds were white and opaque with spongy appearance (Figure 1(a)) and interconnected porous structure. $\beta$-TCP particles were distributed well and homogeneously in the skeleton network of collagen fibrils (Figure 1(b)). The network structure of collagen fibrils was caused by the acidic environment during fabrication. Collagen fibrils are composed of numerous polypeptide chains with carboxyl groups that interconnect via covalent and hydrogen bonds [28]. In dilute acid solutions, $\mathrm{H}^{+}$ ions tend to react with these polypeptide chains, leading to the disassembly of the collagen fibrils and subsequent formation of a network structure. The porosity of the composite scaffolds was around 95\%. The interconnected porous structure may have beneficial effects on cell proliferation and nutritive transportation for applications in bone tissue engineering [29]. In FT-IR spectra (Figure 1(c)), adsorption bands were clearly detected, which corresponded to $\beta$-TCP $\left(\mathrm{PO}_{4}{ }^{3-}: 1627\right.$ and $\left.1572 \mathrm{~cm}^{-1}\right)$ and collagen $\left(\mathrm{COO}^{-}: \sim 1541\right.$, 1448 , and $\left.1399 \mathrm{~cm}^{-1}\right)$. These results further confirmed that $\mathrm{n}-\beta$-TCP/Col scaffolds with an integrated structure can be formed by acid-treated collagen and nanosized $\beta$-TCP particles through chemical bonds.

3.2. Bioactivity of BMSCs in $n-\beta-T C P / C o l$ Scaffolds In Vitro. In the present study, we isolated and purified rabbit BMSCs based on their ability to form adherent clonogenic cell clusters. The isolated BMSCs formed adherent colonies in which the majority of the cells exhibited fibroblastic spindle shapes 


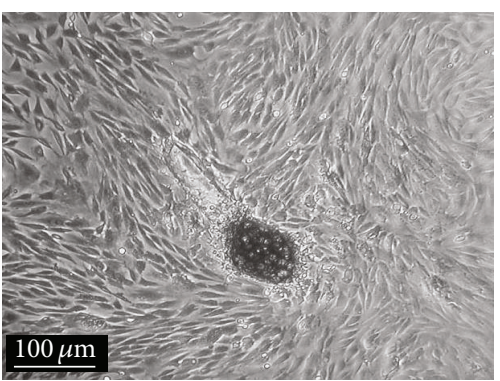

(a)

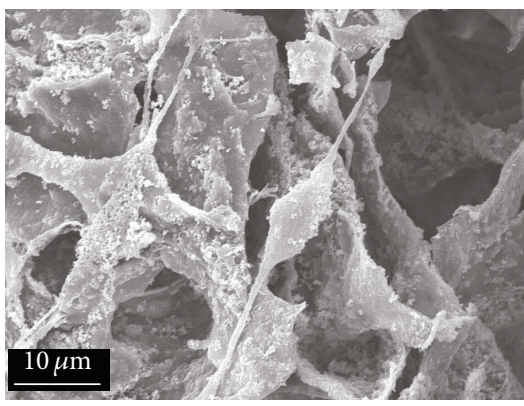

(d)

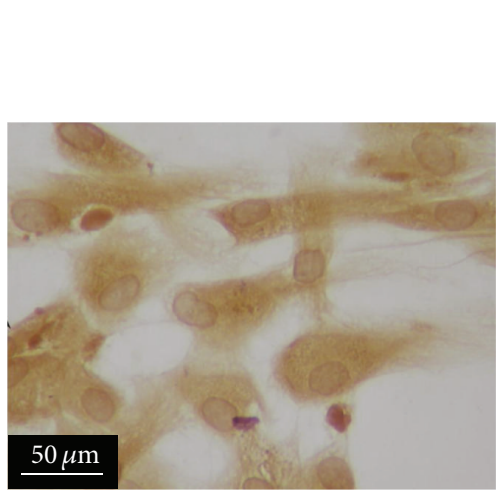

(b)

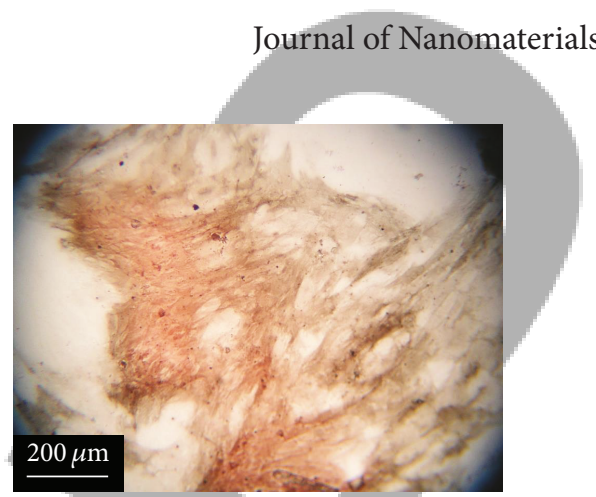

(c)

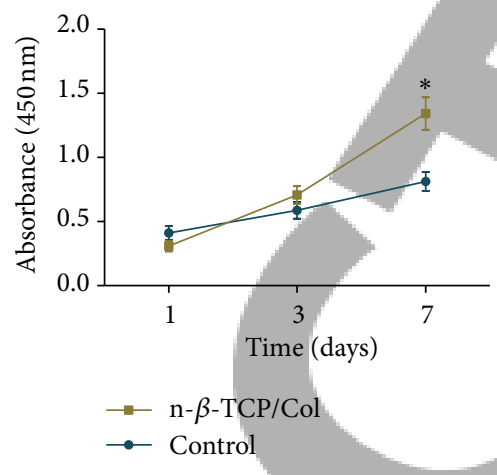

(e)

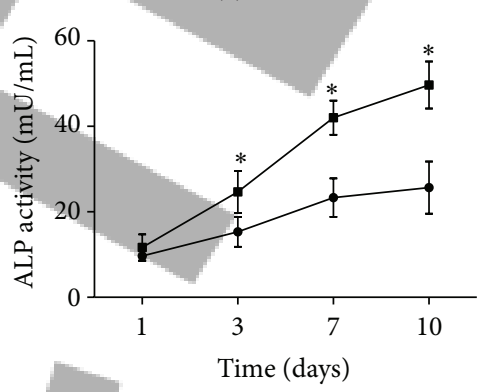

$\mathrm{n}-\beta-\mathrm{TCP} / \mathrm{Col}$

Control

(f)

FIGURE 2: Characterization of rabbit BMSCs and in vitro bioactivity of BMSCs in $\mathrm{n}-\beta$-TCP/Col scaffolds. (a) Micrograph of BMSCs cultured in regular medium for 3 days. (b) CD34 immunohistological staining of BMSCs cultured in regular medium. (c) ALP staining of passage 3 BMSCs cultured in osteogenic medium for 7 days. (d) SEM micrographs of induced BMSCs in the n- $\beta$-TCP/Col scaffolds after 3 days of culture. (e) CCK- 8 assay results of the proliferation of BMSCs in $n-\beta$-TCP/Col scaffolds at various time points. (f) Change of ALP activity in BMSCs in direct contact with the $\mathrm{n}-\beta$-TCP/Col scaffolds after 10 days of incubation in osteogenic medium (BMSCs cultured on tissue culture plates were used as the control).

(Figure 2(a)). Positive staining for CD34 confirmed that the cells displayed a characteristic of BMSCs (Figure 2(b)). To investigate their osteogenic differentiation potential, the cells were cultured in osteogenic medium. After 7 days of induction, the cells showed extensive positive staining for ALP, thereby exhibiting an osteogenic phenotype (Figure 2(c)). Figure 2(d) shows representative phase-contrast micrographs of BMSCs attached to the scaffolds after 3 days of culture. BMSCs mostly showed a fusiform shape, and they were adhered well to the scaffold surfaces and bridged the micropores with their pseudopodia. These features indicated that the scaffold appeared to have no negative effects on cell morphology and viability. The results of cell proliferation assays are shown in Figure 2(e). At day 3, there was no significant difference in the cell number between BMSCs seeded in $n-\beta$ $\mathrm{TCP} / \mathrm{Col}$ scaffolds and cells cultured on tissue culture plates (TCPS). At day 7, there were more cells in the $\mathrm{n}-\beta-\mathrm{TCP} / \mathrm{Col}$ scaffolds compared with those in TCPS. This higher level of cell proliferation may be attributed to the porous structure of the $\mathrm{n}-\beta-\mathrm{TCP} / \mathrm{Col}$ scaffolds, which provides a large surface area for cell spreading [30]. Collectively, these results indicate

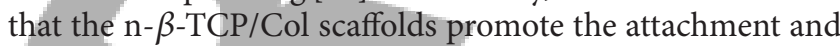
proliferation of BMSCs.

Differentiation of BMSCs is a key process for bone regeneration. In our in vitro experiments, differentiation of the BMSCs into osteoblastic cells in $\mathrm{n}-\beta$-TCP/Col scaffolds was quantitatively measured by ALP activity (Figure 2(f)). ALP activity is an early marker that identifies osteoblastic cells and is considered as an important factor in bone differentiation and mineralization [31,32]. After 1 day of osteogenic stimulation, low ALP activity was detected, indicating few BMSCs had differentiated into osteoblastic cells. However, after further stimulation, the level of ALP activity increased substantially $(P<0.05)$. These results indicate that $\mathrm{n}-\beta-\mathrm{TCP} /$ Col scaffolds improve the osteogenic differentiation ability of BMSCs.

3.3. Osteogenesis of BMSCs Hybridized with $n-\beta-T C P / C o l$ Scaffolds In Vivo. To evaluate the osteogenic potential and effects of autologous BMSCs in $\mathrm{n}-\beta$-TCP/Col scaffolds in vivo, a rabbit mandible defect was selected to assess the performance of the biomaterial [23]. A critical-sized defect model is imperative for the evaluation of biomaterials in bone defect repair $[33,34]$. Therefore, we established a critical defect $\left(10 \times 8 \times 3 \mathrm{~mm}^{3}\right)$ in the mandible of each rabbit. During the experiment, all rabbits remained in good health and did not show any wound complications.

Figure 3 shows macroscopic images of the implantation. At 4 weeks after implantation, the surface of defects in the only scaffolds group was partially filled with callus bone, while a large proportion of the residual scaffold was observed in the defect site (Figure 3(a1)). In contrast, a larger area was filled with callus bone in defects implanted 


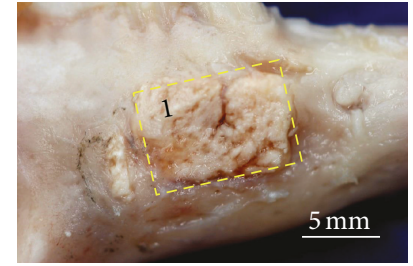

(a1)

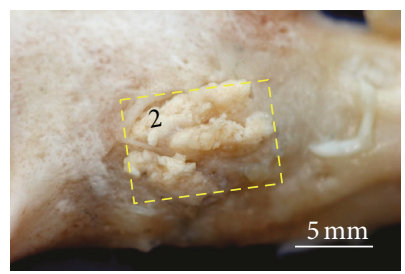

(b1)

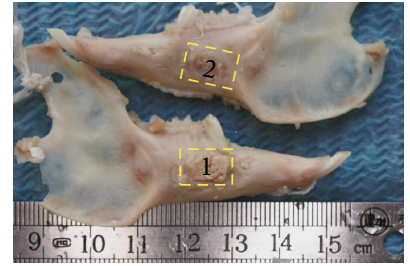

(a)

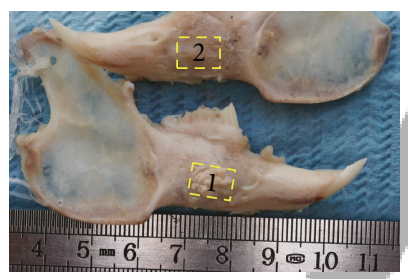

(b)

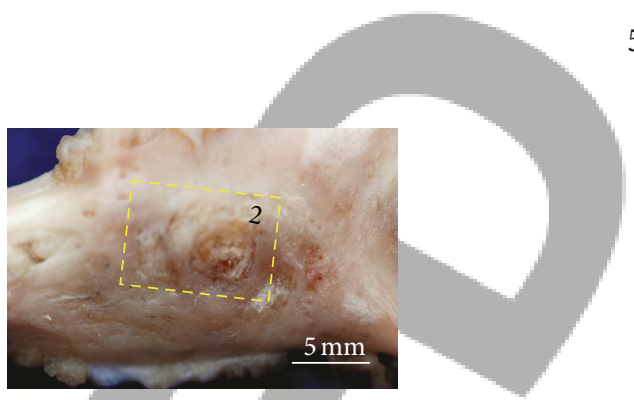

(a2)

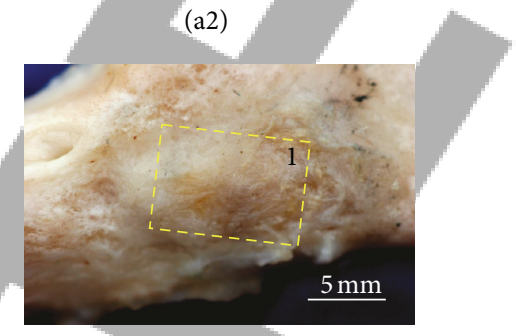

(b2)

Figure 3: Macroscopic images of the implantation sites at 4 weeks (a) and 8 weeks (b). (a1 and b1) n- $\beta$-TCP/Col scaffolds and (a2 and b2) BMSC/scaffold constructs.

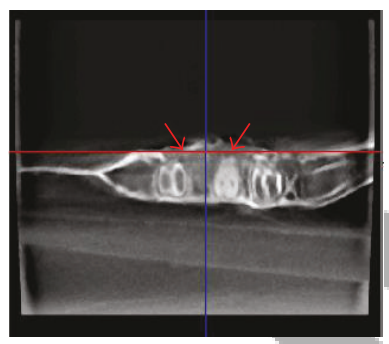

(a)

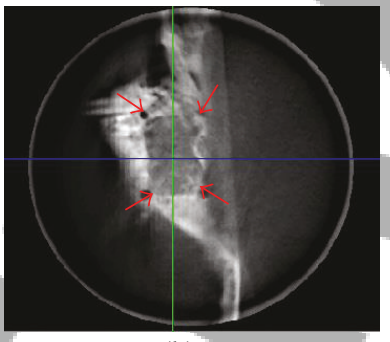

(b)

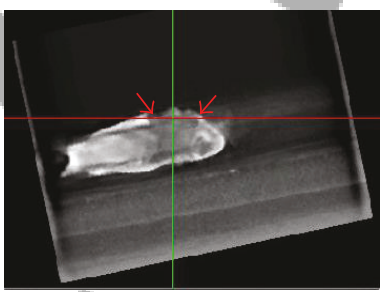

(c)

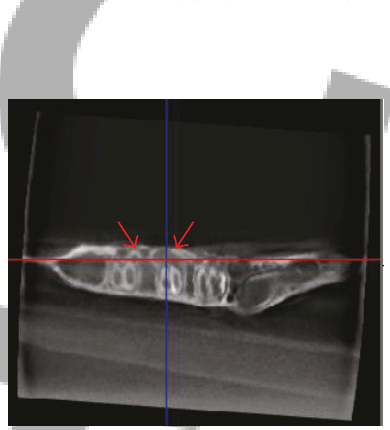

(d)

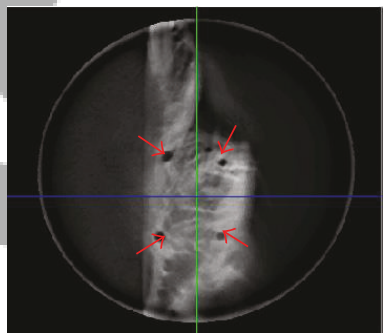

(e)

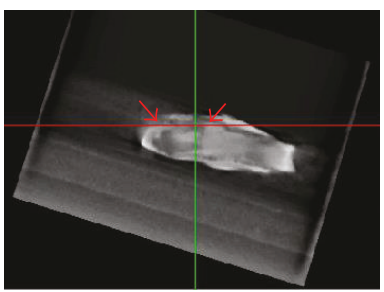

(f)

FIGURE 4: CBCT images of rabbit mandible defects with $\mathrm{n}-\beta$-TCP/Col scaffolds $(\mathrm{a}-\mathrm{c})$ or BMSC/scaffold constructs (d-f) at 8 weeks after implantation. (a, d) Coronal section, (b, e) sagittal section, $(c, f)$ and horizontal section. Red arrows denote the surgical site.

with BMSC/scaffold constructs, and the boundary between scaffolds and host bone was unclear because of active osteogenesis in the implantation area (Figure 3(a2)). At 8 weeks after implantation, the wounds were well healed and there were few differences in the hardness and appearance of newly formed bone and normal osseous tissues in the BMSC/scaffold construct group (Figure 3(b2)). In contrast, in the only scaffolds group, there was still a proportion of the scaffolds retained in the defects (Figure 3(b1)), which was confirmed by CBCT scanning (Figure 4 ). These results indicate that BMSC/scaffold constructs provide a better osteogenic outcome than that of pure scaffolds. 


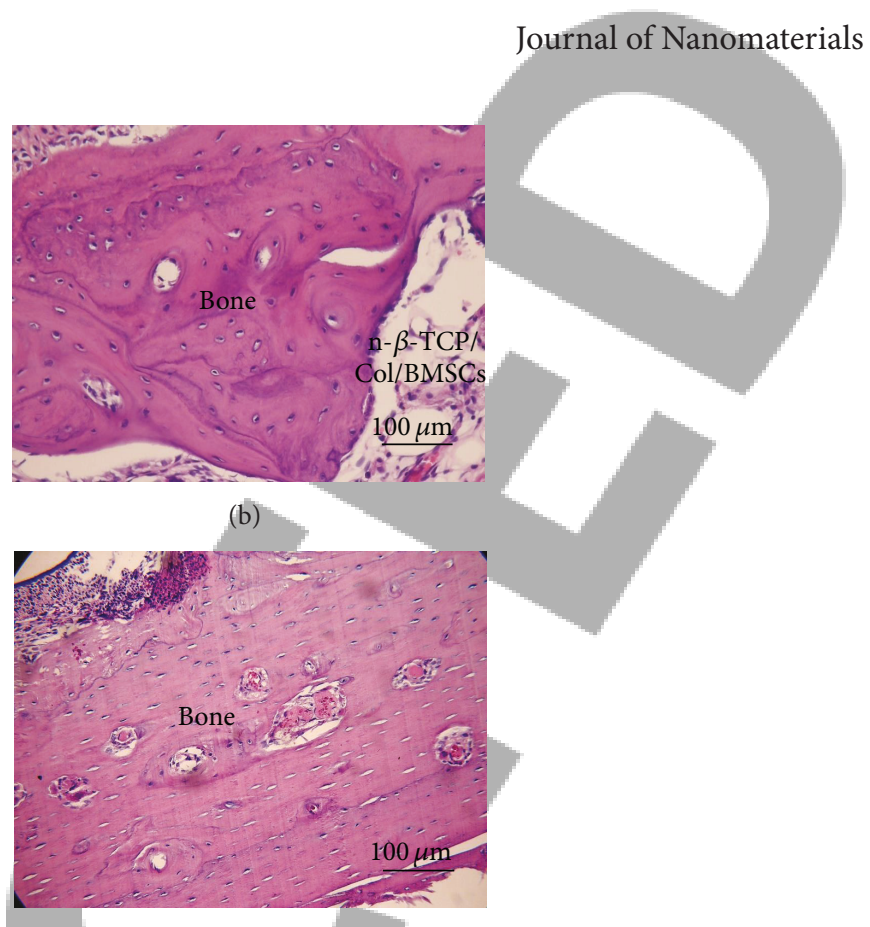

(c)

(d)

FIGURE 5: Histological images of mandible defects at 4 weeks $(a, b)$ and 8 weeks $(c, d)$ after implantation of n- $\beta$-TCP/Col scaffolds (a, c) and BMSC/scaffold constructs (b, d).

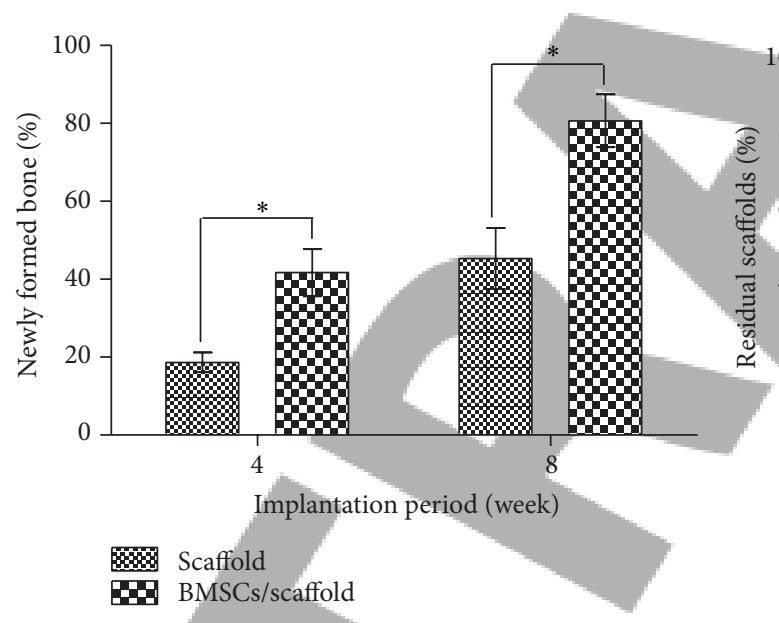

(a)

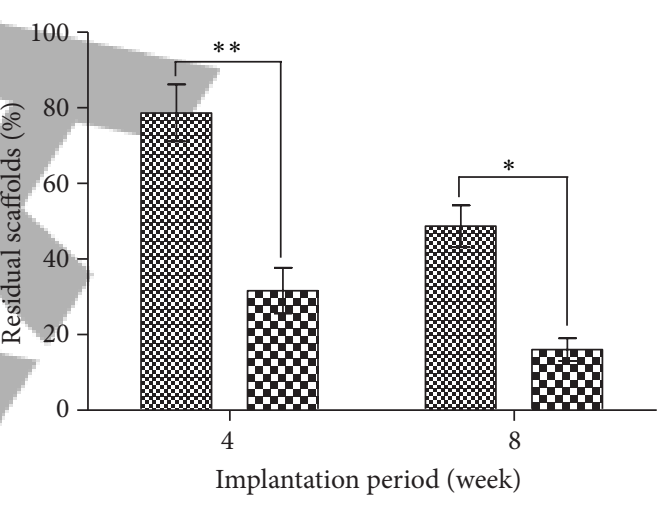

$\$$ Scaffold

BO BMSCs/scaffold

(b)

FIGURE 6: Newly formed bone area (a) and residual scaffolds area (b) in rabbit mandible defects at 4 and 8 weeks after implantation.

Figure 5 shows the histological results of mandible defect repair, indicating different trends of bone regeneration and scaffolds degradation from the two groups. In the scaffolds only group, the defect was mainly occupied by an abundance of residual scaffolds with a dense structure at 4 weeks after implantation (Figure 5(a)). In addition, residual scaffolds were present in the defect despite the obvious appearance of newly formed bone at 8 weeks after implantation (Figure 5(c)). In contrast, BMSC/scaffold constructs exhibited not only high biocompatibility but also faster and more efficient osteogenesis in the defect area. At 4 weeks after implantation, a small amount of residual scaffolds with a loose structure was observed in the center of defects that were mostly dominated by newly formed bone (Figure 5(b)).
Moreover, osteoblasts (an indication of bone formation) were observed at the interface between the scaffolds and bone. From these results, seeded BMSCs might differentiate into osteoblast in vivo and probably account for the improved bone formation, which is consistent with previous report [23]. At 8 weeks after implantation, there was almost complete restoration of the bone defects with little residual materials (Figure 5(d)). The histological observations were confirmed by quantitative analyses of the percentages of newly formed bone and residual scaffolds (Figure 6).

These results indicate that incorporation of in vitro expanded autologous BMSCs into the porous $n-\beta-T C P / C o l$ scaffolds enhances the efficiency of bone regeneration, especially during the initial period after implantation. Our 
findings can be explained by the fact that the interconnected macropores of the $n-\beta-T C P / C o l$ scaffolds facilitate cell infiltration into the interior of the scaffolds [35]. When cultureexpanded autologous BMSCs at a relatively high cell density are preseeded into scaffolds, BMSCs differentiate towards osteoblasts during the early period after implantation, which increases the rate of bone regeneration. In summary, the $\mathrm{n}-\beta$-TCP/Col scaffolds hybridized with culture-expanded autologous BMSCs presented not only high biocompatibility but also faster and more effective bone defect restoration.

\section{Conclusions}

In the present study, we have successfully prepared porous $\mathrm{n}-\beta$-TCP/Col composite scaffolds with an interconnected porous structure and $\beta$-TCP particles that are homogeneously distributed in the skeleton network of the collagen fibrils. Our results show that $\mathrm{n}-\beta$-TCP/Col scaffolds exhibit high biocompatibility in vitro. Introduction of autologous BMSCs into the scaffolds dramatically enhances the efficiency of critical-sized bone defects restoration, especially during the initial stage after implantation. Collectively, these results suggest that culture-expanded autologous BMSCs in $n-\beta$ $\mathrm{TCP} / \mathrm{Col}$ scaffolds have the potential to be applied in bone tissue engineering.

\section{Conflict of Interests}

The authors declare that there is no conflict of interests regarding the publication of this paper.

\section{Authors' Contribution}

Xuehui Zhang and Mingming Xu equally contributed to this paper.

\section{Acknowledgments}

This work was supported by the National Basic Research Program of China (2012CB933900), Beijing Municipal Science and Technology Commission (Z121100005212007), the National Natural Science Foundation of China (81171000), and the National High Technology Research and Development Program of China (2012AA022501). Additionally, the authors also would like to acknowledge the support of the Peking University-Tsinghua University Joint Center for Life Sciences.

\section{References}

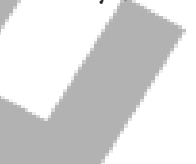

[1] J. O. Hollinger and J. C. Kleinschmidt, "The critical size defect as an experimental model to test bone repair materials," The Journal of Craniofacial Surgery, vol. 1, no. 1, pp. 60-68, 1990.

[2] Y. Liu, G. Wu, and K. De Groot, "Biomimetic coatings for bone tissue engineering of critical-sized defects," Journal of the Royal Society Interface, vol. 7, supplement 5, pp. S631-S647, 2010.

[3] T. Liu, G. Wu, D. Wismeijer, Z. Gu, and Y. Liu, "Deproteinized bovine bone functionalized with the slow delivery of BMP-2 for the repair of critical-sized bone defects in sheep," Bone, vol. 56, no. 1, pp. 110-118, 2013.

[4] M. H. Mankani, S. A. Kuznetsov, R. M. Wolfe, G. W. Marshall, and P. G. Robey, "In vivo bone formation by human bone marrow stromal cells: reconstruction of the mouse calvarium and mandible," Stem Cells, vol. 24, no. 9, pp. 2140-2149, 2006.

[5] M. Nishikawa, A. Myoui, H. Ohgushi, M. Ikeuchi, N. Tamai, and $\mathrm{H}$. Yoshikawa, "Bone tissue engineering using novel interconnected porous hydroxyapatite ceramics combined with marrow mesenchymal cells: quantitative and three-dimensional image analysis," Cell Transplantation, vol. 13, no. 4, pp. 367-376, 2004.

[6] M. C. Kruyt, C. Persson, G. Johansson, W. J. A. Dhert, and J. D. De Bruijn, “Towards injectable cell-based tissue-engineered bone: the effect of different calcium phosphate microparticles and pre-culturing," Tissue Engineering, vol. 12, no. 2, pp. 309317, 2006.

[7] A. Minamide, M. Yoshida, M. Kawakami et al., "The use of cultured bone marrow cells in type I collagen gel and porous hydroxyapatite for posterolateral lumbar spine fusion," Spine, vol. 30, no. 10, pp. 1134-1138, 2005.

[8] J. Yuan, L. Cui, W. J. Zhang, W. Liu, and Y. Cao, "Repair of canine mandibular bone defects with bone marrow stromal cells and porous $\beta$-tricalcium phosphate," Biomaterials, vol. 28, no. 6, pp. 1005-1013, 2007.

[9] G. Liu, L. Zhao, W. Zhang, L. Cui, W. Liu, and Y. Cao, "Repair of goat tibial defects with bone marrow stromal cells and $\beta$ tricalcium phosphate," Journal of Materials Science, vol. 19, no. 6, pp. 2367-2376, 2008.

[10] D. W. Hutmacher, "Scaffolds in tissue engineering bone and cartilage," Biomaterials, vol. 21, no. 24, pp. 2529-2543, 2000.

[11] A. J. Salgado, O. P. Coutinho, and R. L. Reis, "Bone tissue engineering: state of the art and future trends," Macromolecular Bioscience, vol. 4, no. 8, pp. 743-765, 2004.

[12] K. Kurashina, H. Kurita, Q. Wu, A. Ohtsuka, and H. Kobayashi, "Ectopic osteogenesis with biphasic ceramics of hydroxyapatite and tricalcium phosphate in rabbits," Biomaterials, vol. 23, no. 2, pp. 407-412, 2002.

[13] J. Dong, T. Uemura, Y. Shirasaki, and T. Tateishi, "Promotion of bone formation using highly pure porous $\beta$-TCP combined with bone marrow-derived osteoprogenitor cells," Biomaterials, vol. 23, no. 23, pp. 4493-4502, 2002.

[14] K.-Y. Chen, C.-M. Chung, Y.-S. Chen, D.-T. Bau, and C.-H. Yao, "Rat bone marrow stromal cells-seeded porous gelatin/tricalcium phosphate/oligomeric proanthocyanidins composite scaffold for bone repair," Journal of Tissue Engineering and Regenerative Medicine, vol. 7, no. 9, pp. 708-719, 2013.

[15] X. Liu and P. X. Ma, "Polymeric scaffolds for bone tissue engineering," Annals of Biomedical Engineering, vol. 32, no. 3, pp. 477-486, 2004.

[16] S. J. Hollister, "Porous scaffold design for tissue engineering," Nature Materials, vol. 4, no. 7, pp. 518-524, 2005.

[17] G. Wei and P. X. Ma, "Structure and properties of nanohydroxyapatite/polymer composite scaffolds for bone tissue engineering," Biomaterials, vol. 25, no. 19, pp. 4749-4757, 2004.

[18] H. H. K. Xu and C. G. Simon Jr., "Fast setting calcium phosphate-chitosan scaffold: mechanical properties and biocompatibility," Biomaterials, vol. 26, no. 12, pp. 1337-1348, 2005.

[19] B. M. B. Brkovic, H. S. Prasad, M. D. Rohrer et al., "Betatricalcium phosphate/type I collagen cones with or without a barrier membrane in human extraction socket healing: clinical, histologic, histomorphometric, and immunohistochemical 
evaluation," Clinical Oral Investigations, vol. 16, no. 2, pp. 581590, 2012.

[20] J. E. Mate-Sanchez de Val, P. Mazon, J. L. Guirado et al., "Comparison of three hydroxyapatite/beta-tricalciumphosphate/collagen ceramic scaffolds: an in vivo study," Journal of Biomedical Materials Research A. In press.

[21] T. Matsuno, T. Nakamura, K.-I. Kuremoto et al., "Development of $\beta$-tricalcium phosphate/collagen sponge composite for bone regeneration," Dental Materials Journal, vol. 25, no. 1, pp. 138144, 2006.

[22] C. Zou, W. Weng, X. Deng et al., "Preparation and characterization of porous $\beta$-tricalcium phosphate/collagen composites with an integrated structure," Biomaterials, vol. 26, no. 26, pp. 5276-5284, 2005.

[23] H. Wang, Y. Li, Y. Zuo, J. Li, S. Ma, and L. Cheng, "Biocompatibility and osteogenesis of biomimetic nano-hydroxyapatite/polyamide composite scaffolds for bone tissue engineering," Biomaterials, vol. 28, no. 22, pp. 3338-3348, 2007.

[24] Y. Li, W. Weng, K. Cheng et al., "Preparation of amorphous calcium phosphate in the presence of poly(ethylene glycol)," Journal of Materials Science Letters, vol. 22, no. 14, pp. 1015-1016, 2003.

[25] C. Zou, W. Weng, X. Deng et al., "Preparation and characterization of porous $\beta$-tricalcium phosphate/collagen composites with an integrated structure," Biomaterials, vol. 26, no. 26, pp. 5276-5284, 2005.

[26] X. Zhang, Q. Cai, H. Liu et al., "Osteoconductive effectiveness of bone graft derived from antler cancellous bone: an experimental study in the rabbit mandible defect model," International Journal of Oral \& Maxillofacial Surgery, vol. 41, no. 11, pp. 1330 1337, 2012.

[27] Y. Sawada, A. Hokugo, Y. Yang et al., "A novel hydroxyapatite ceramic bone substitute transformed by ostrich cancellous bone: characterization and evaluations of bone regeneration activity," Journal of Biomedical Materials Research B, vol. 98, no. 2, pp. 217-222, 2011.

[28] M.J. Beckman, K.J. Shields, and R. F. Diegelmann, "Collagen," in Encyclopedia of Biomaterials and Biomedical Engineering, G. E. Wnek and G. L. Bowlin, Eds., pp. 324-334, Marcel Dekker, New York, NY, USA, 2004.

[29] S. P. Bruder and B. S. Fox, "Tissue engineering of bone: cell based strategies," Clinical Orthopaedics and Related Research, no. 367, supplement, pp. S68-S83, 1999.

[30] H.-W. Kim, H.-E. Kim, and V. Salih, "Stimulation of osteoblast responses to biomimetic nanocomposites of gelatin-hydroxyapatite for tissue engineering scaffolds," Biomaterials, vol. 26, no. 25, pp. 5221-5230, 2005.

[31] R. Marom, I. Shur, R. Solomon, and D. Benayahu, "Characterization of adhesion and differentiation markers of osteogenic marrow stromal cells," Journal of Cellular Physiology, vol. 202, no. 1, pp. 41-48, 2005.

[32] U. Stucki, J. Schmid, C. F. Hämmerle, and N. P. Lang, “Temporal and local appearance of alkaline phosphatase activity in early stages of guided bone regeneration. A descriptive histochemical study in humans," Clinical Oral Implants Research, vol. 12, no. 2, pp. 121-127, 2001.

[33] K. Kurashina, H. Kurita, A. Kotani, H. Takeuchi, and M. Hirano, "In vivo study of a calcium phosphate cement consisting of $\alpha$-tricalcium phosphate/dicalcium phosphate dibasic/tetracalcium phosphate monoxide," Biomaterials, vol.18, no. 2, pp. 147-151, 1997.
[34] M. Kikuchi, Y. Koyama, T. Yamada et al., "Development of guided bone regeneration membrane composed of $\beta$-tricalcium phosphate and poly (L-lactide-co -glycolide- $c o$ - $\varepsilon$ - caprolactone) composites," Biomaterials, vol. 25, no. 28, pp. 5979-5986, 2004.

[35] H. Guo, J. Su, J. Wei, H. Kong, and C. Liu, "Biocompatibility and osteogenicity of degradable Ca-deficient hydroxyapatite scaffolds from calcium phosphate cement for bone tissue engineering," Acta Biomaterialia, vol. 5, no. 1, pp. 268-278, 2009.
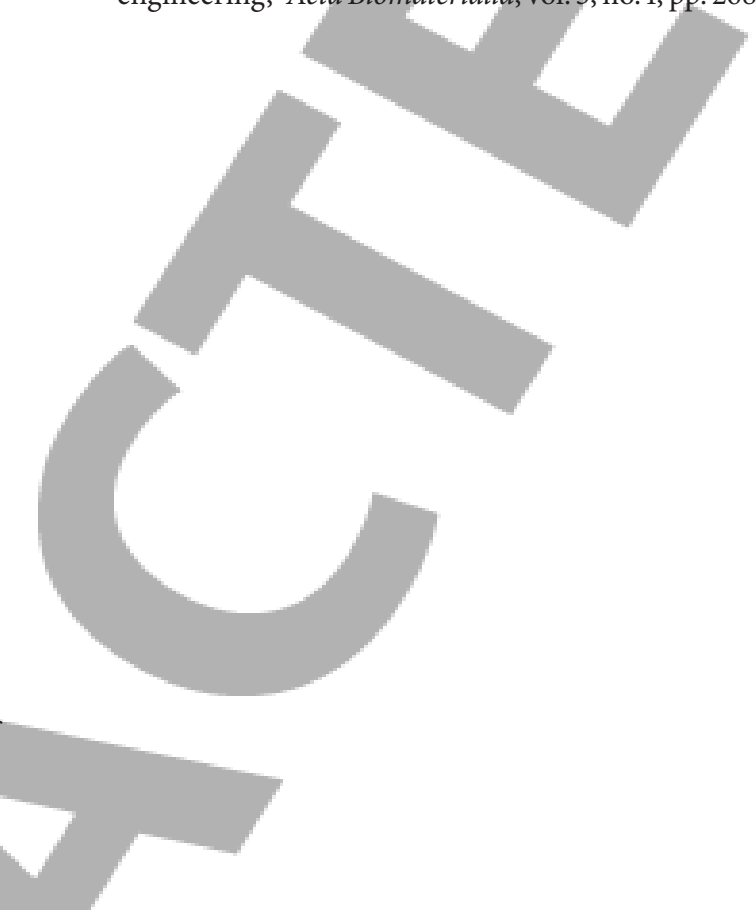